Full Paper

\title{
Functional role of surface layer proteins of Lactobacillus acidophilus L-92 in stress tolerance and binding to host cell proteins
}

\author{
Taketo WAKAI ${ }^{1 *}$, Chie KANO ${ }^{1}$, Harma KARSENS ${ }^{2}$, Jan $\mathrm{KOK}^{2}$ and Naoyuki YAMAMOTO ${ }^{3}$ \\ ${ }^{1}$ Core Technology Laboratories, Asahi Quality and Innovations, Ltd., 5-11-10 Fuchinobe, Chuo-ku, Sagamihara-shi, Kanagawa, Japan \\ ${ }^{2}$ Department of Molecular Genetics, Groningen Biomolecular Sciences and Biotechnology Institute, University of Groningen, \\ Linnaeusborg, Nijenborgh 7, Groningen, The Netherlands \\ ${ }^{3}$ School of Life Science and Technology, Tokyo Institute of Technology, 4259 Nagatsuta-cho, Midori-ku, Yokohama-shi, Kanagawa, \\ Japan
}

Received January 29, 2020; Accepted August 8, 2020; Published online in J-STAGE September 17, 2020

\begin{abstract}
Lactobacillus acidophilus surface layer proteins (SLPs) self-assemble into a monolayer that is non-covalently bound to the outer surface of the cells. There they are in direct contact with the environment, environmental stressors and gut components of the host in which the organism resides. The role of $L$. acidophilus SLPs is not entirely understood, although SLPs seem to be essential for bacterial growth. We constructed three $L$. acidophilus L-92 strains, each expressing a mutant of the most abundant SLP, SIpA. Each carried a 12-amino acid c-myc epitope substitution at a different position in the protein. A strain was also obtained that expressed the SlpA paralog SlpB from an originally silent $\operatorname{SlpB}$ gene. All four strains behaved differently with respect to growth under various stress conditions, such as the presence of salt, ox gall or ethanol, suggesting that SlpA affects stress tolerance in L. acidophilus L-92. Also, the four mutants showed differential in vitro binding ability to human host cell proteins such as uromodulin or dendritic cell (DC)-specific intercellular adhesion molecule-3 grabbing nonintegrin (DC-SIGN). Furthermore, co-culture of murine immature DCs with a mutant strain expressing one of the recombinant SIpA proteins changed the concentrations of the cytokines IL-10 and IL-12. Our data suggest that SIpA and SIpB of $L$. acidophilus participate in bacterial stress tolerance and binding to uromodulin or DC-SIGN, possibly leading to effective immune-modification.
\end{abstract}

Key words: Lactobacillus acidophilus, surface layer proteins, stress tolerance, probiotics, immune regulation

\section{INTRODUCTION}

Lactobacillus acidophilus is one of the most studied and consumed probiotic bacteria because of its high ability to survive in digestive juice, to bind to gut epithelial cells and to modulate host immune function $[1,2]$. Our group has studied the effect of $L$. acidophilus L-92 mainly on immune function. Oral administration of L. acidophilus L-92 [3] has been reported to improve symptoms of atopic dermatitis [4-6], pollen allergy [7] and perennial allergic rhinitis [8] in humans and mice. In addition, oral intake of the strain protected against influenza infection in the mouse and human [9, 10]. Strain L-92 has immune-regulating activities in mice, such as the regulation of cytokine production, suppression of antigenspecific IgE, induction of apoptosis of antigen-stimulated $\mathrm{T}$ cells and induction of regulatory T cells [11-13].
L. acidophilus produces surface layer proteins (SLPs) that form a self-assembled monolayer on the outer surface of the cells [14]. Given this location, these proteins might be important for contact with the environment [15]. L. acidophilus can potentially produce 3 SLPs, SlpA, SlpB and SlpX, in addition to expressing various surface layer-associated proteins (SLAPs) [16]. SlpA is the most abundant SLP, as SlpX is only expressed at low amounts and SlpB is not expressed because the gene does not have a functional promoter [17]. An L. acidophilus NCFM slpX knockout strain showed both a lower survival rate under specific growth conditions and lower binding to mucin [18]. These observations are indicative of the importance of (certain) SLPs for environmental stress tolerance and the binding to components of host cells. An L. acidophilus NCFM mutant carrying an $\operatorname{slp} A$ knockout insertion expressed SlpB, which was caused by

*Corresponding author. Taketo Wakai (E-mail: taketo.wakai@asahi-qi.co.jp)

(Supplementary material: refer to PMC https://www.ncbi.nlm.nih.gov/pmc/journals/2480/)

(C2021 BMFH Press

This is an open-access article distributed under the terms of the Creative Commons Attribution Non-Commercial No Derivatives (by-nc-nd) License. (CC-BY-NC-ND 4.0: https://creativecommons.org/licenses/by-nc-nd/4.0/) 
an inversion between the originally silent $\operatorname{slp} B$ gene and $\operatorname{slp} A$, resulting in $\operatorname{slp} B$ being under the control of the $\operatorname{slp} A$ promoter in this strain $[19,20]$. These results indicate that $L$. acidophilus cells need to express at least SlpA or SlpB on their surface in order to grow and survive and that SLPs might be very important or even essential in L. acidophilus.

SlpA is one of the key components in L. acidophilus binding to host proteins and further immune-modulation. L. acidophilus CP23, which has a relatively low level of SLPs on its surface, shows lower adhesion activity to caco-2 cells and less IL-12 release from DCs than L. acidophilus strains possessing more SLPs [21, 22]. Immune regulation by $L$. acidophilus NCFM SlpA operates via its binding to one of the C-type lectin receptors, the specific intracellular adhesion molecule-3 grabbing nonintegrin homolog-related 3 (SIGNR3) in the mouse and dendritic cell (DC)-specific intercellular adhesion molecule-3 grabbing non-integrin (DC-SIGN) in humans $[19,23]$. In addition, the uromodulin expressed in microfold (M) cells selectively binds to SlpA on L. acidophilus L-92 cells [24]. Binding leads to selective incorporation of the SlpA-expressing bacteria into the $\mathrm{M}$ cells and to their effective delivery to DCs.

In addition to SLPs, several SLAPs have been shown to affect the organism's binding activity to host components and immune-modulating activity $[16,25,26]$. Deletion of PrtX (encoded by LBA1578) was reported to increase adhesion of the L. acidophilus NCFM mutant to mucin and fibronectin [25], while on the other hand, deletion of another SLAP, LBA0191, decreased the adhesion to both human proteins [26]. When murine DCs were exposed to cells lacking PrtX, the concentrations of the pro-inflammatory interleukins IL-6 and IL-12, that of the antiinflammatory interleukin IL-10, and the IL-10/IL-12 ratio were increased compared with those of the parent strain. Increase of the IL-10/IL-12 ratio suggests a pro-inflammatory status. The LBA1029 deletion mutant caused lower induction of TNF- $\alpha$ in murine DCs than its parent [16].

In the present study, we constructed four genetically modified L. acidophilus L-92 SlpA mutants by inserting a c-myc epitope at different positions in the protein and investigated the roles of certain regions in SlpA on both bacterial stress tolerance and binding to host proteins such as uromodulin and DC-SIGN.

\section{MATERIALS AND METHODS}

\section{Bacterial strains and growth conditions}

The L. acidophilus L-92 used was from the stock collection of Asahi Group Holdings Ltd. L. acidophilus strains were grown in de Man, Rogosa and Sharpe (MRS) medium (BD, Franklin Lakes, NJ, USA) at $37^{\circ} \mathrm{C}$. In general, 0.5 to $5 \%$ of a culture was inoculated in fresh medium and incubated overnight at $37^{\circ} \mathrm{C}$ without shaking. Lactococcus lactis and Escherichia coli were used for cloning purposes. L. lactis was cultured at $30^{\circ} \mathrm{C}$ in Difco M17 medium (BD) containing $0.5 \%$ glucose. E. coli was cultivated in Luria Bertani (LB) medium (Formedium, Norfolk, UK) at $37^{\circ} \mathrm{C}$ with shaking. The media were supplemented with $5 \mu \mathrm{g} / \mathrm{mL}$ chloramphenicol $(\mathrm{Cm}$; for Lactobacillus and Lactococcus) or $10 \mu \mathrm{g} / \mathrm{mL} \mathrm{Cm}$ (for E. coli), and when needed, they were further supplemented with $5 \mu \mathrm{g} / \mathrm{mL}$ erythromycin (Ery; for Lactobacillus and Lactococcus) or $150 \mu \mathrm{g} / \mathrm{mL}$ Ery (for E. coli). Strains, plasmids and primers used in this study are listed in Supplementary Tables 1 and 2. Cloning, transformation and mutagenesis strategies are described in detail in the Supplementary Materials and Methods online.

\section{SDS-PAGE and Western blotting}

Tricin sodium dodecyl sulfate- $10 \%$ polyacrylamide gel electrophoresis (SDS-PAGE) and Coomassie brilliant blue (CBB) staining were performed as described previously [27]. Cells cultured overnight at $37^{\circ} \mathrm{C}$ in MRS (with $1 \mu \mathrm{g} / \mathrm{mL}$ Ery only for SAr2) were washed and resuspended in PBS to adjust the $\mathrm{OD}_{600}$ to 4.8 . Cell solutions $(30 \mu \mathrm{L})$ were mixed with $10 \mu \mathrm{L}$ of Laemmli sample buffer (Bio-Rad Laboratories) and then boiled for $10 \mathrm{~min}$, after which $10 \mu \mathrm{L}$ of the samples were applied to the SDS-polyacrylamide gel. Proteins were blotted onto a polyvinylidene fluoride (PVDF) membrane (Bio-Rad Laboratories), after which immune detection of recombinant SlpA containing the c-myc epitope was carried out with an antic-myc mouse monoclonal antibody (Thermo Fisher Scientific), horseradish peroxidase (HRP)-conjugated anti-mouse IgG antibody (Thermo Fisher Scientific) and chemiluminescent detection kit (ECL Prime kit, Bio-Rad Laboratories) according to manufacturers' manuals. Samples from some protein bands were purified from gel fragments and analyzed by nano LC/MS/ MS (Applied Biosystems) at JPROteomics (Miyagi, Japan), as described previously [28]. The Mascot software was used to perform mass data acquisition.

\section{SLP and SLAP isolation}

SLPs and SLAPs were isolated essentially according to a previous report [16]. Cells grown overnight in MRS were harvested by centrifugation at $8,000 \mathrm{~g}$ for $5 \mathrm{~min}$ and washed twice with PBS. All subsequent steps were conducted at $4{ }^{\circ} \mathrm{C}$. Cells were resuspended in a $1 / 20$ volume of $5 \mathrm{M} \mathrm{LiCl}$, gently agitated for $15 \mathrm{~min}$, and then centrifuged at $20,000 \mathrm{~g}$ for $30 \mathrm{~min}$. The supernatants were dialyzed in a 10,000-Da membrane overnight against water. After dialysis, the solutions were centrifuged at $20,000 \mathrm{~g}$ for $30 \mathrm{~min}$. The precipitates were then resuspended in $1 \mathrm{M} \mathrm{LiCl}$ and incubated at $4^{\circ} \mathrm{C}$ for $15 \mathrm{~min}$, after which the suspensions were centrifuged at 20,000 g for $10 \mathrm{~min}$. For SLP fractions, the precipitates were then washed three times with cold water and resuspended in water or $10 \%$ SDS. For SLAP fractions, the supernatants after centrifugation in the $1 \mathrm{M} \mathrm{LiCl}$ incubation step were dialyzed, centrifuged, washed and resuspended in water or $10 \%$ glycerol.

\section{Fluorescence microscopy and flow cytometry}

The c-myc epitope was detected with antibodies, fluorescence microscopy and flow cytometry basically as described previously $[29,30]$. Cells were grown overnight in MRS at $37^{\circ} \mathrm{C}$, harvested and washed with PBS. The washed cells were resuspended in PBS containing $0.25 \%$ bovine serum albumin (BSA) and the primary antibody (50-fold diluted mouse anti-c-myc antibody) and incubated on ice for $30 \mathrm{~min}$. The cells were then washed twice with PBS and resuspended in PBS with $0.25 \%$ BSA and the 100 fold diluted secondary antibody (goat anti-mouse IgG secondary antibody, FITC, Thermo Fisher Scientific) and incubated on ice for $30 \mathrm{~min}$. Subsequently, the cells were washed with PBS and analyzed by fluorescence microscopy and flow cytometry. Fluorescent levels in approximately 50,000 cells were measured with a BD FACSCanto flow cytometer (BD Biosciences, Franklin Lakes, NJ, USA) using a 488-nm argon laser. Raw data were 
collected using the FACSDiva software (BD Biosciences). WinMDI 2.9 was used for data analysis (http://en.bio-soft.net/ other/WinMDI.html). Microscopy images were taken with a DeltaVision microscope (Applied Precision, Issaquah, WA, USA) equipped with a $2300-\mathrm{W}$ xenon light source, $100 \times$ bright field objective, and a GFP filter set (Chroma, Bellows Falls, VT, USA; excitation at $470 / 40 \mathrm{~nm}$ and emission at $525 / 50 \mathrm{~nm}$ ).

\section{Determination of growth and stress tolerance}

L. acidophilus strains were cultured overnight in MRS medium. The overnight culture of each strain was diluted in fresh MRS medium with or without $0.005 \%$ SDS, $0.3 \%$ ox gall, $5 \%$ ethanol (EtOH) or $2 \%$ sodium chloride $(\mathrm{NaCl})$ to an $\mathrm{OD}_{600}$ of around 0.03 and incubated at $37^{\circ} \mathrm{C}$ in the wells of a 96-well microtiter plate with a transparent lid in a Powerscan HT microplate reader (DS Pharma Biomedical, Osaka, Japan). The $\mathrm{OD}_{600}$ was measured every hour after $10 \mathrm{sec}$ of shaking.

\section{DC-SIGN and uromodulin binding assay}

L. acidophilus cells grown in MRS for $20 \mathrm{hr}$ were harvested and washed with the same volume of PBS twice and then resuspended in the same volume of $0.1 \%$ trisodium citrate. The cell suspension was heated at $95^{\circ} \mathrm{C}$ for $10 \mathrm{~min}$ to kill the cells. Subsequently, they were washed with PBS and resuspended in PBS while adjusting the cell concentration to the desired density for the binding and co-culturing assays. The binding ability of L. acidophilus strains to DC-SIGN and uromodulin was analyzed using ELISA as described previously [19]. A recombinant human DC-SIGN/ CD209 Fc chimera protein (R\&D Systems, Minneapolis, MN, USA) and recombinant mouse uromodulin Fc chimera protein [24] were used. Fifty microliters of the heat-killed bacterial cells $\left(7.5 \times 10^{7}\right.$ cells $\left./ \mathrm{mL}\right)$ were coated on NUNC 96-well MaxiSorp plates (Thermo Fisher Scientific) and incubated overnight at $4{ }^{\circ} \mathrm{C}$. The plates were blocked with $1 \%$ of BSA in PBS for 1.5 hr. DC-SIGN $(5 \mu \mathrm{g} / \mathrm{mL})$ or uromodulin $(5 \mu \mathrm{g} / \mathrm{mL})$ in PBS was added, and the plates were then incubated for $1 \mathrm{hr}$ with gentle shaking. Bound protein was detected with an HRP-conjugated goat anti-human IgG-Fc fragment antibody (Bethyl Laboratories, Montgomery, TX, USA) and TMB One Component HRP Microwell Substrate (BioFX Laboratories, Owings Mills, MD, USA) according to the manufacturers' protocols. The statistical significance of differences between the parent strain and each variant strain was calculated using the Dunnett test in the IBM SPSS Statistics software (IBM Corp., Armonk, NY, USA).

\section{Incubation of dendritic cells with recombinant \\ L. acidophilus strains}

Animal experiments were approved by the Institutional Animal Experiment Committee (Application No. 16-28-01) of Asahi Group Holdings, Ltd. on December 2, 2016, and performed in 2017 at the animal facility of Asahi Group Holdings, Ltd. Female BALB/c mice (5 weeks old) were purchased from Japan SLC. Murine immature dendritic cells were prepared from bone marrow cells as described previously [31]. Bone marrow from mice femurs was flushed using cold PBS. Cells were treated with Tris-buffered ammonium chloride to lyse erythrocytes. The remaining cells were cultured in RPMI 1640 complete medium plus $10 \%$ fetal bovine serum (FBS) with mouse granulocytemacrophage colony-stimulating factor (GM-CSF; $20 \mathrm{ng} / \mathrm{mL}$ ) in NUNC six-well tissue culture plates (Thermo Fisher Scientific).
After $6 \mathrm{hr}$ of incubation, non-adherent cells were removed, and the remaining cells were cultured in fresh RPMI 1640 complete medium plus $10 \%$ FBS containing GM-CSG for 7 days. Every other day, cultures were fed the fresh medium containing GMCSF. On day 7, cells were harvested and, after confirmation that more than $90 \%$ were CD11c positive, they were treated with heat-killed $L$. acidophilus cells at a DC-to- $L$. acidophilus ratio of $1: 10$ or $1: 1$. After $24 \mathrm{hr}$ of incubation, the supernatants were sampled to measure cytokines with a Bio-Plex Pro Mouse Th1 7-plex kit (Bio-Rad Laboratories) according to the manufacturer's protocol. DCs $\left(5 \times 10^{5}\right)$ were incubated with surface marker monoclonal antibodies for $30 \mathrm{~min}$ at $4^{\circ} \mathrm{C}$, washed extensively with BD Stain Buffer (BD Biosciences) and analyzed using a FACSCanto flow cytometer (BD Biosciences). At least $10^{4}$ gated events per condition were acquired. The statistical significance of differences between the parent strain and each mutant was calculated using 2-way ANOVA in SPSS and the post hoc paired t-test with Bonferroni correction.

\section{RESULTS}

\section{Construction of $\mathrm{L}$. acidophilus L-92 expressing recombinant SlpA}

As the $\operatorname{slp} A$ gene seems to be essential for L. acidophilus L-92 and cannot be deleted (data not shown), we decided to make mutant strains expressing recombinant forms of SlpA. A stretch of ten amino acid residues in non-conserved regions in SlpA was replaced with 12 amino acid residues containing the c-myc epitope as an expression tag. The recombinant genes were integrated at the $\operatorname{slp} A$ locus using double crossover gene replacement recombination (DCO; Table 1). From a total of 10 different recombinant $\operatorname{slp} A$ genes that we attempted to make, only 3 could be obtained that were expressed (Table 1). Two mutant proteins, SlpAr2 and SlpAr3, carried the c-myc epitope in an area in the SlpA protein that is predicted to be surface exposed [29, 32]. The other two, SlpAr1 and SlpAr4, carried the substitution in a structurally unspecified region. In total, four mutants were obtained in L. acidophilus L-92 $\Delta u p p$, a strain in which the upp gene was deleted to enable using the upp-based counterselection system [18]. DCO, as confirmed by PCR results showing replacement of the original $\operatorname{slp} A$ gene by the recombinant copy of $\operatorname{slp} A$, was attained in three cases (strains SAr1, SAr4 and SAr3; $\mathrm{SAr} 3$ renamed to SB3; see below). In one strain, designated SAr2, only single crossover (SCO) could be obtained such that both the original slp $A$ gene and the recombinant copy, slpAr2, were present in the chromosome. The SAr2 strain was used for further studies, as excision of the integrated plasmid, required for exchange of the $\operatorname{slp} A$ genes, was repeatedly unsuccessful. The $\operatorname{slp} A$ regions in the chromosomes of the three DCO mutants were sequenced, and the presence and identity of the recombinant $\operatorname{slp} A$ version was confirmed in two (SAr1 and SAr4). However, in one (SAr3, designated SB3 hereafter), the $\operatorname{slp} B$ gene was present at the $\operatorname{slp} A$ locus, while the recombinant $\operatorname{slp} A r 3$ gene was at the silent $\operatorname{slp} B$ locus, suggesting that homologous recombination had occurred between the adjacent $\operatorname{slp} A$ and $\operatorname{slp} B$ genes. L. acidophilus L-92 $\operatorname{slp} B$ is a paralog of $\operatorname{slp} A$; it is located in the opposite orientation at around $3 \mathrm{kbp}$ downstream of $\operatorname{slp} A$ (Supplementary Fig. 1). The $\operatorname{sip} B$ gene has two regions of identity with $\operatorname{sip} A$. One area of around $300 \mathrm{bp}$ is located in the $5^{\prime}$-UTR and the start of the coding regions; the other (approximately $400 \mathrm{bp}$ ) is present at the end 
Table 1. Status summary of recombinant L. acidophilus L-92 slpA mutants

\begin{tabular}{|c|c|c|c|c|c|c|c|}
\hline Strain & Gene & $\begin{array}{l}\text { Position of AA } \\
\text { substitution }^{\mathrm{a}}\end{array}$ & $\begin{array}{l}\text { Size of AA } \\
\text { substitution } \\
(\mathrm{AA})^{\mathrm{b}}\end{array}$ & AA sequence ${ }^{c}$ & Status $^{\mathrm{d}}$ & Western $^{\mathrm{e}}$ & Microscope $^{f}$ \\
\hline SAr1 & $\operatorname{slp} A r 1$ & 61 & $-10+12$ & svsa-EQKLISEEDLAS-aiag & $\mathrm{DCO}$ & Detected & Detected \\
\hline SAr2 & $\begin{array}{l}\text { slpAr2 } \\
\text { slpAr3g }\end{array}$ & 94 & $-10+12$ & tanl-EQKLISEEDLAS-aags & $\begin{array}{c}\mathrm{SCO} \\
\mathrm{DCO}^{\mathrm{g}}\end{array}$ & Detected & Detected \\
\hline SB3 & $(\operatorname{slp} B+)$ & 167 & $-10+12$ & nvst-EQKLISEEDLAS-asln & $(\mathrm{SlpB}+)$ & ND & - \\
\hline SAr4 & $\operatorname{slp} A r 4$ & 246 & $-10+12$ & aqya-EQKLISEEDLAS-teda & $\mathrm{DCO}$ & Detected & ND \\
\hline
\end{tabular}

a: Substituted amino acid (AA) position relative to the initiator methionine of SlpA. (See Supplementary Fig. 2 for entire sequences of SlpA and SlpB.)

b: Number of AAs deleted $(-)$ and inserted $(+)$ to incorporate the c-myc epitope at the indicated position.

c: Inserted sequence containing the c-myc epitope is indicated in 1-letter code with capital letters; flanking AA sequences of the original SlpA are indicated in small letters.

$\mathrm{d}$ : Status of integration of the recombinant slpA gene in the L. acidophilus chromosome.

e: Detected, positive in Western hybridization using anti-c-myc antibodies; ND, not detected (Fig. 1).

f: Results of fluorescence microscopy analysis of whole cells treated with the c-myc antibody. ND, not detected; -, not tested (Fig. 2).

$\mathrm{g}$ : Due to homologous recombination, the $\operatorname{slp} A r 3$ and $\operatorname{slp} B$ genes have swapped places in the chromosome (see Supplementary Fig. 1 and the text for details).

\section{Western hybridization CBB staining}

$(\mathrm{kDa})$

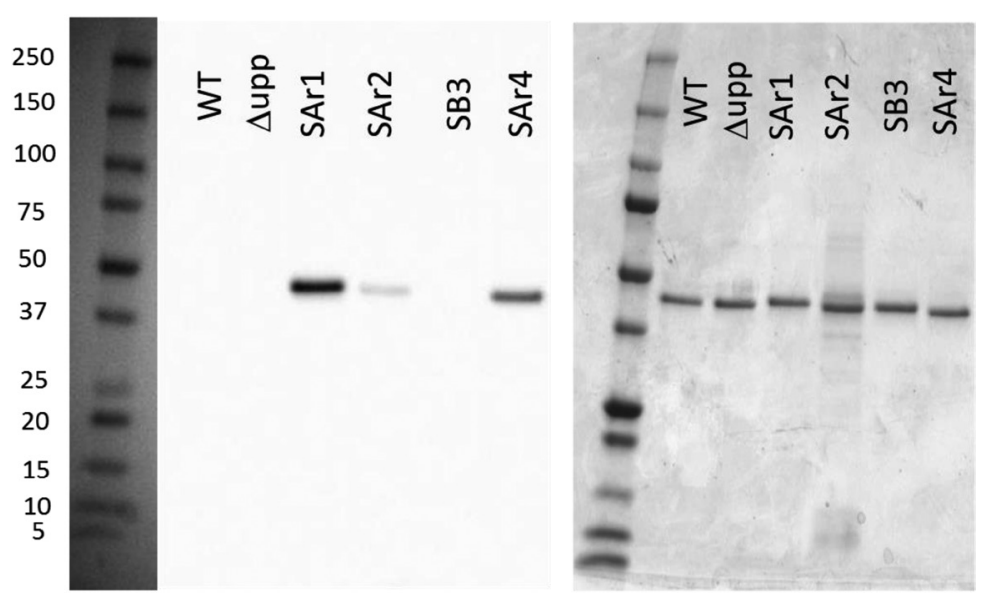

Fig. 1. Production of recombinant SlpA by L. acidophilus. Left, Western hybridization using anti-c-myc antibodies of SLPs from overnight-grown cells; right, Coomassie brilliant blue (CBB)-stained gel loaded with the same amount of cell suspension. WT, strain L. acidophilus L-92. See Table 1 for descriptions of the other samples. The molecular weight of mature SlpA is $43.6 \mathrm{kDa}$. The protein size marker is the same in both panels.

of the coding regions of both genes. As a result of the presumed homologous recombination event, the entire $\operatorname{slp} B$ gene is under the control of the slpA promoter in strain SB3. The $s l p A r 3$ gene is not expressed, as the $\operatorname{slp} B$ locus does not carry an active promoter [33]. The four mutants were all used in further experiments.

\section{Recombinant SlpA proteins are expressed in $\mathrm{L}$. acidophilus}

Washed whole-cell suspensions of all four recombinant strains and their parent were boiled and subjected to SDS-PAGE, after which the gels were blotted and examined by Western analysis using anti-c-myc antibodies. As expected, all strains produced proteins in the size range of SlpA of approximately $43 \mathrm{kDa}$ (Fig. 1). The recombinant and original SlpA proteins could not be distinguished on the gel, as they had almost the same sizes. Strain SB3 also produced such a protein, but it did not react with the c-myc antibody. This protein was a surface layer protein that was abundant on SB3 cells, and it was identified as SlpB through peptide mass fingerprinting (see below). Furthermore, SCO strain SAr2 showed a weak antibody response, suggesting that the amount of recombinant SlpA-c-myc protein (SlpAr2) produced by this strain was less than that made by SAr1 and SAr4. Most likely, strain SAr2 produces a mixture of SlpA and recombinant SlpAr2. The DCO strains SAr1 and SAr4 express their respective recombinant SlpA proteins of the expected sizes, each of which reacts with the c-myc antibody.

\section{The c-myc epitopes in recombinant SlpA are surface exposed}

To examine whether the parts in SlpA carrying the c-myc epitope substitutions are at the outside of the cells and could thus be in contact with host cell components, their accessibility for the c-myc antibody in whole cells was investigated. Cells of the three recombinant SlpA-expressing strains were first coated with a mouse anti-c-myc antibody and then with a FITC-labeled antimouse IgG antibody. The strains SAr1 and SAr2 reacted with the antibodies and were detectable by both fluorescence microscopy and flow cytometry (Fig. 2). In other words, the c-myc epitopes in the surface layer formed by these recombinant SlpA proteins are accessible from the outside. Strain SAr4, like its parent L. acidophilus L-92 4 upp, did not react with the antibodies; apparently, the c-myc epitope is shielded in this strain. 

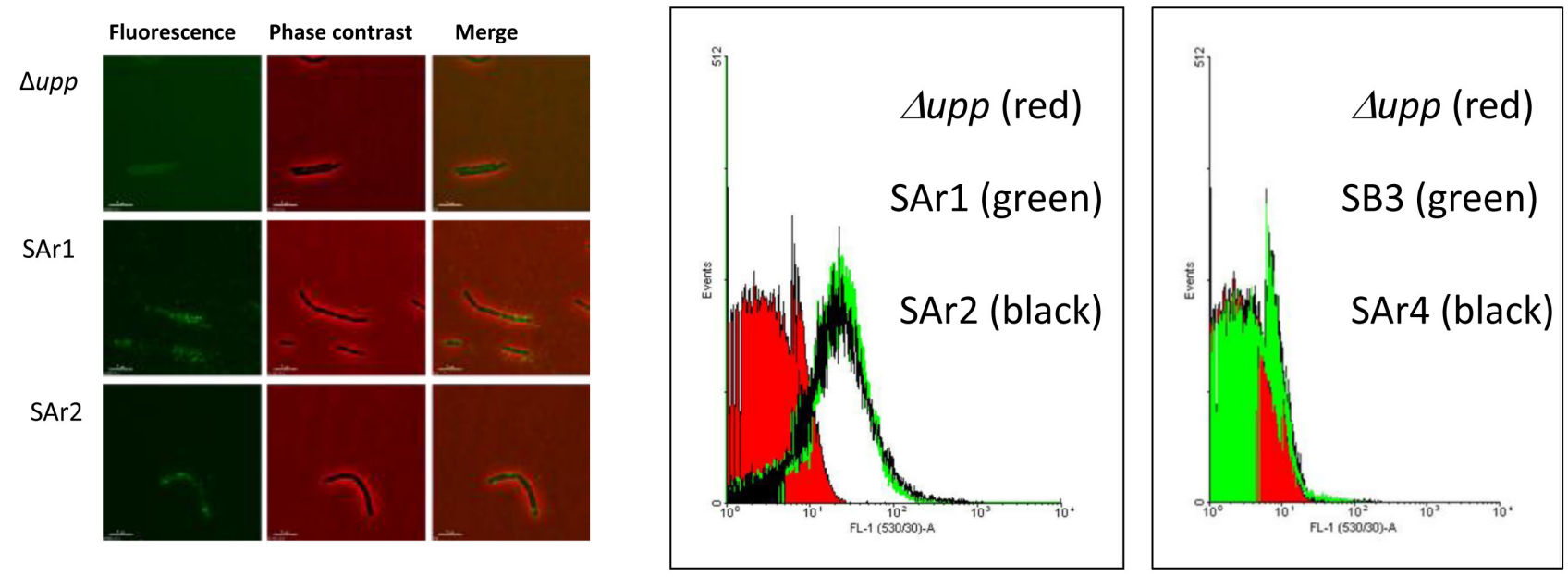

Fig. 2. Microscopy (left) and flow cytometric analyses (center and right) of L. acidophilus strains expressing recombinant SlpA. Cells grown overnight were washed and treated first with a mouse anti-c-myc antibody and then with a FITC-labeled anti-mouse IgG antibody. Typical micrographs are shown. White bar, $1 \mu \mathrm{m}$. For the FACS analyses, 50,000 events were recorded, and they are shown in the histograms, which show fluorescence intensity on the $\mathrm{x}$-axis and the number of events (cell count) on the $\mathrm{y}$-axis.

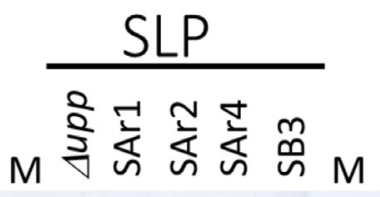

(kDa)

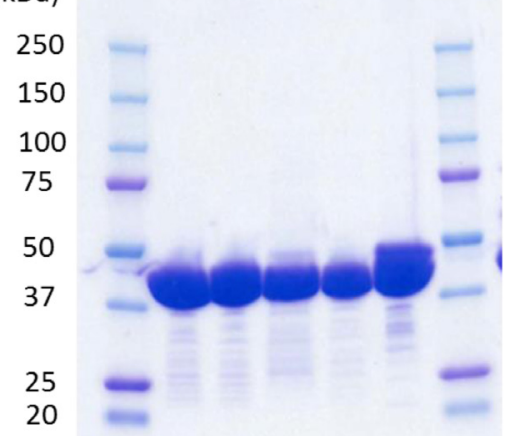

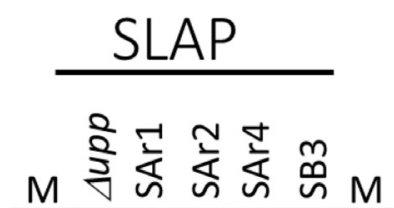

Fig. 3. Surface layer proteins (SLPs) and surface layer-associated proteins (SLAPs) extractable from $L$. acidophilus strains. Left, SDS (4-20\%)-PAGE of SLP fractions. Right, SLAP fractions. Surface-attached proteins were extracted from cells grown overnight, using $5 \mathrm{M} \mathrm{LiCl}$. The precipitated proteins after dialysis of $5 \mathrm{M} \mathrm{LiCl}$ extract were washed with $1 \mathrm{M} \mathrm{LiCl}$. The resulting precipitated proteins and soluble proteins in the $1 \mathrm{M} \mathrm{LiCl}$ washing solution were SLP and SLAP fractions, respectively. M, protein marker (the same in both gels). The sizes of protein markers are indicated in the left margin. Description of the strains/samples (Table 1).

\section{Surface layer-associated proteins}

Surface layer-associated proteins have previously been identified on L. acidophilus NCFM cells. They have also been shown to affect the organism's binding activity to host components and immune-modulating activity $[16,25,26]$. As SLAPs are thought to be associated with SLPs [16], the amino acid changes in the recombinant SlpAs might affect the SLAP patterns of the L. acidophilus mutants studied here. The SLPs and SLAPs of the recombinant strains were isolated by $\mathrm{LiCl}$ extraction and analyzed by SDS-PAGE. The SLP fractions of L. acidophilus L-92 $\Delta$ upp and three of the recombinant strains showed a single intense band of a protein of the size of SlpA, while two bands were present in the extract of strain SB3 (Fig. 3). The proteins in the latter two bands were isolated from the SDS-polyacrylamide gel and identified with peptide mass fingerprinting. The larger band was identified as SlpX, while the protein in the smaller band, running to the same position as SlpA in the other three samples, was SlpB. The SLAP fractions of the parent and all four recombinant strains showed rather similar protein banding patterns and intensities, although a number of small differences were apparent. A few additional bands were visible, and some were relatively abundant in strains SAr2 and SB3 compared with the parent strain. For example, bands at around $21 \mathrm{kDa}$ and $17 \mathrm{kDa}$ were visible only in $\mathrm{SAr} 2$ and SB3. These results suggest that the recombinant SlpA protein SlpAr2 and either SlpB or overexpressed SlpX affect the pattern and abundances of SLAPs. 
Table 2. Growth characteristics of L. acidophilus strains

\begin{tabular}{lccccc}
$\mu$ Max $\left(\mathrm{h}^{-1}\right)$ & & & & & \\
\hline & Supp & SAr1 & SAr2 & SB3 & SAr4 \\
\hline MRS & 0.65 & 0.56 & 0.56 & 0.68 & 0.51 \\
+ EtOH & 0.41 & 0.36 & 0.35 & 0.44 & 0.23 \\
+ NaCl & 0.45 & 0.38 & 0.41 & 0.51 & 0.43 \\
+ Oxgal & 0.62 & 0.6 & 0.4 & 0.57 & 0.54 \\
+ SDS & 0.51 & 0.54 & 0.43 & 0.62 & 0.5 \\
\hline
\end{tabular}

\begin{tabular}{lccccc} 
Lag time (hr) & & & & & \\
\hline & Supp & SAr1 & SAr2 & SB3 & SAr4 \\
\hline MRS & 3.4 & 3.7 & 2.7 & 3.2 & 3.2 \\
+ EtOH & 4.8 & 4.5 & 3.7 & 3.4 & 4.6 \\
+ NaCl & 7.3 & 8.8 & 6.3 & 5.1 & 6.7 \\
+ Oxgal & 4.4 & 4.3 & 2.9 & 2.5 & 4.2 \\
+ SDS & 3.3 & 3 & 2.1 & 2.3 & 3.1 \\
\hline
\end{tabular}

The data are extracted from the curves in Fig. 3.

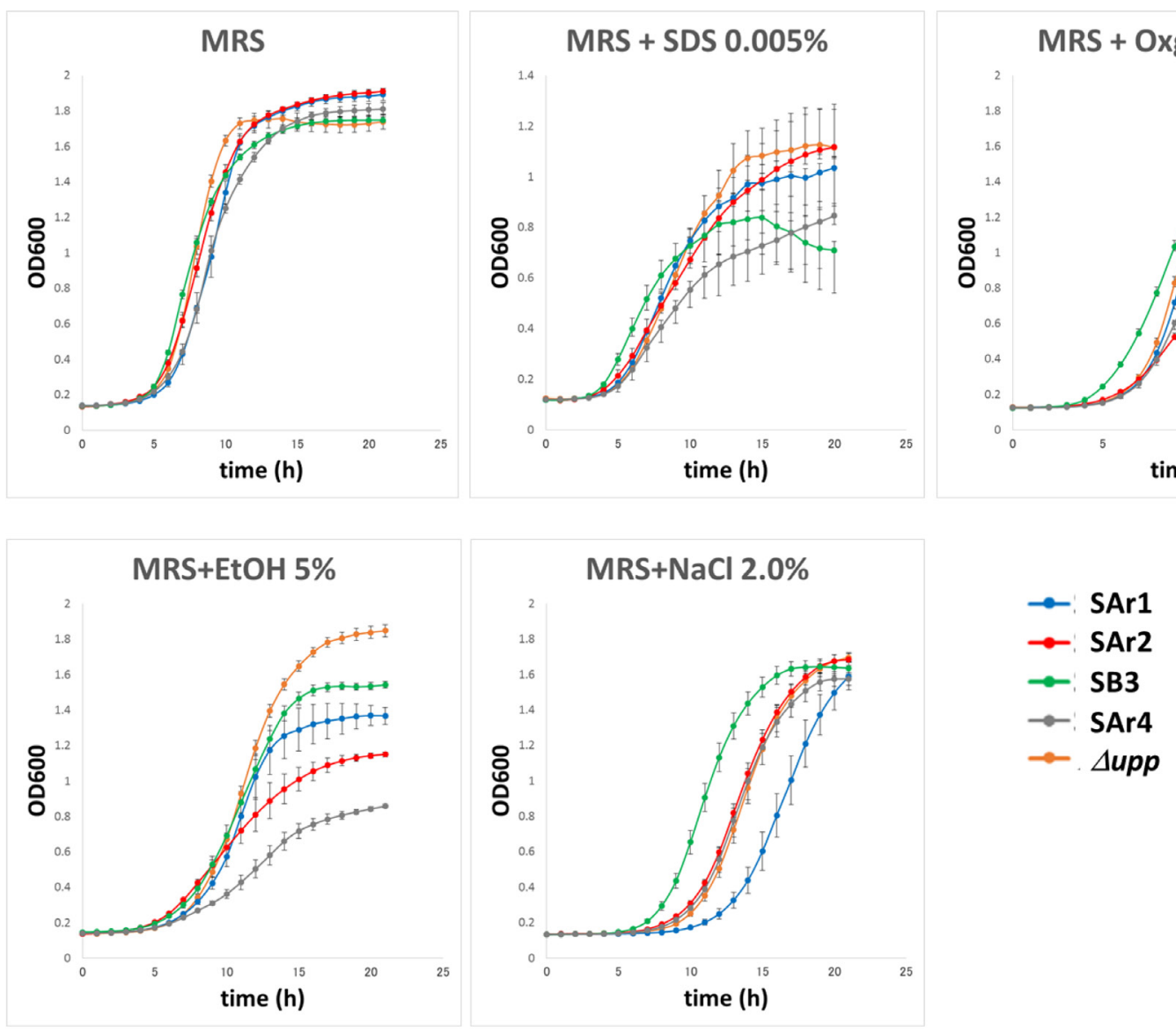

Fig. 4. Growth and stress tolerance of L. acidophilus recombinant strains. The various strains were grown in MRS medium with or without the indicated chemical in $96-$ well microtiter plates at $37^{\circ} \mathrm{C}$. Data are shown as the mean of triplicate wells. The experiment was repeated three times with similar results. Error bars indicate the standard deviation.

\section{Growth and stress tolerance of $\mathrm{L}$. acidophilus $L-92$ expressing mutant SlpA}

When the growth of L. acidophilus L-92 $\triangle$ upp and its mutants was examined in the nutritionally rich MRS medium, no differences were observed between the growth rates of the strains (Fig. 4 and Table 2). It has been suggested that SLPs have a role in tolerance to environmental stressors such as detergent, salt and alcohol. Indeed, each mutant showed strain-specific stress tolerance patterns when exposed to various stress conditions. The lag time of SAr1 in MRS with $\mathrm{NaCl}$ was longer than that of the parent, while growth was comparable in other media (data not shown). The growth rate of SAr2 was greatly decreased in ox gallcontaining MRS broth, and the final optical density was lower than that of the parent. The lag time of SB3, the strain expressing SlpB, was shorter than that of its parent under all stress conditions tested. Strain SAr4 grew slower in ethanol-containing medium than the parent.

\section{Binding of DC-SIGN and uromodulin to L. acidophilus L-92 and its mutants}

Next, ELISA was performed to evaluate the interaction of the three recombinant SlpA-expressing strains and the strain expressing SlpB and SlpX with two specific host proteins: mouse uromodulin $\mathrm{Fc}$ chimera protein and recombinant human DCSIGN/CD209 Fc chimera protein (Fig. 5). The binding of DCSIGN to heat-killed SAr1 and SAr4 cells was slightly decreased 

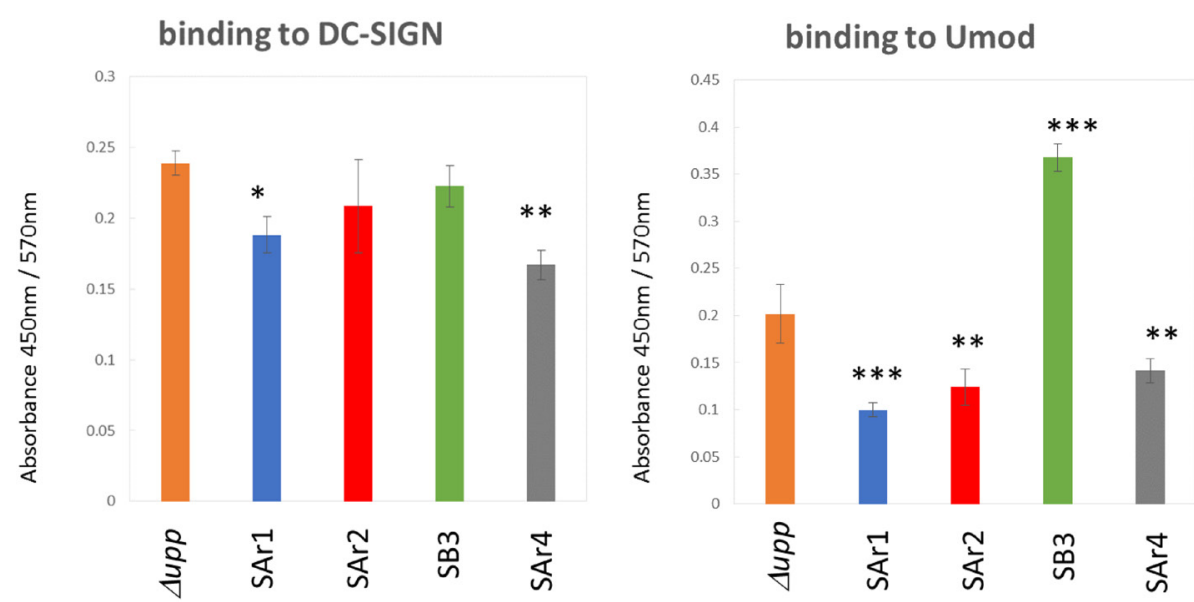

Fig. 5. In vitro binding of L. acidophilus strains to host proteins. An enzyme-linked immunosorbent assay (ELISA) was used to measure binding of the cells with DC-SIGN-Fc or uromodulin (Umod)-Fc chimera proteins. Heat-killed exponential-phase cells were coated on 96-well plates. DC-SIGN-Fc or Umod-Fc was added to the plates. Bound protein was detected with a horseradish peroxidase (HRP)-conjugated goat anti-human IgG-Fc fragment antibody and tetramethylbenzidine (TMB) by measuring absorbance. Data are the means of triplicate wells. The experiment was repeated three times with similar results. Error bars indicate the standard deviation. Statistical significance was calculated between the parent strain and each variant strain (Dunnett test). ${ }^{*} \mathrm{p}<0.05 ; * * \mathrm{p}<0.01 ; * * \mathrm{p}<0.001$

relative to the parent. Compared with the parent strain, binding of uromodulin to all three recombinant SlpA-producing mutants was significantly decreased, while it bound better to strain SB3. This result suggests that expression of SlpB and SlpX in strain SB3 does not affect the binding of DC-SIGN but instead strengthens the binding of uromodulin. The strains SAr1 and SAr4 showed decreased binding of both host proteins. The two strains express a recombinant SlpA variant in which the c-myc epitope is predicted to be, respectively, either surface exposed or not. Apparently, changes in the identity of SLPs as well as the structure of one of them, SlpA, can all affect binding of host proteins such as DCSIGN and uromodulin.

\section{Cytokine production from DCs is changed by $\mathrm{L}$. acidophilus L-92 SAr2}

Heat-killed recombinant L. acidophilus L-92 cells were incubated with immature dendritic cells (iDCs) derived from murine bone marrow to examine their role in cytokine production and maturation. Expression of three maturation markers, CD80, CD86 and MHCII, in iDCs after 24 hours of treatment with each of the mutant strains was not changed compared with that after treatment with the parent (data not shown), suggesting that they do not affect DC maturation. The concentration of 6 cytokines measured was not changed when iDCs were incubated with strain SAr1, SB3 or SAr4 compared with when they were incubated with the parent (Fig. 6). However, when the iDCs were incubated with strain SAr2, the concentrations of IL-10 and IL-12 were decreased compared with when the iDCs were incubated with the parent. These results may suggest that an alteration of SLPs and/ or SLAPs on the mutant strain SAr2 changed DC signaling.

\section{DISCUSSION}

The four mutant L. acidophilus strains constructed here either express a recombinant version of the SlpA protein or a combination of SlpB and SlpX. SlpB is normally not expressed, as it is encoded by a silent gene without a functional promoter. The strains were used to demonstrate the importance of SLPs, of which at least one should be expressed on the surface of $L$. acidophilus, in bacterial stress tolerance and host protein binding, two critical parameters in the modulation of the host immune system.

$\mathrm{SlpB}$ is paralogous to SlpA, with $58 \%$ amino acid identity (Supplementary Fig. 2). Expression of SlpB (and SlpX, which is overexpressed in strain SB3) might allow the bacteria to grow better than when they would only express slpAr3, or the latter could even be lethal. Expression of small amounts of SlpX, next to SlpA, was detected in L. acidophilus strains L-92 (unpublished data) and NCFM [16], suggesting that all three recombinant strains obtained here might also express some SlpX but that the amount could not be determined by SDS-PAGE. Abundant expression of SlpX in the presence of SlpB, observed here for L. acidophilus SB3, was also reported in L. acidophilus $\Delta$ slpA expressing SlpB [18], indicating that SlpB cannot substitute for SlpA function without SlpX. How SlpX expression is upregulated is not clear yet. The promoter region of $\operatorname{slp} X$ in SB3 is identical to that of the parent. SlpX overexpression might be caused by transcriptional regulation responding to some (stress) condition when SlpB is expressed instead of SlpA, but clearly, the mechanism of overexpression of SlpX and the role of this minor SLP need further attention.

Previous studies have shown that some SLP or SLAP gene deletion strains of $L$. acidophilus, such as knockout strains of $\operatorname{slp} X$ and aggregation-promoting factor gene $a p f$, altered bacterial stress tolerance $[18,34]$. Our data showed that the stress tolerance of L. acidophilus L-92 expressing recombinant SlpAr1, SlpAr2, SlpAr4 or SlpB/X was changed compared with the parent strain. The strains expressing SlpAr1, SlpAr2 and SlpAr4 are more sensitive to sodium chloride, ox gall or ethanol, respectively. In addition, expression of recombinant SlpAr2 or SlpB/X affected SLAP protein patterns. The data suggest that SLPs play a crucial role in growth under certain stress conditions either directly or 


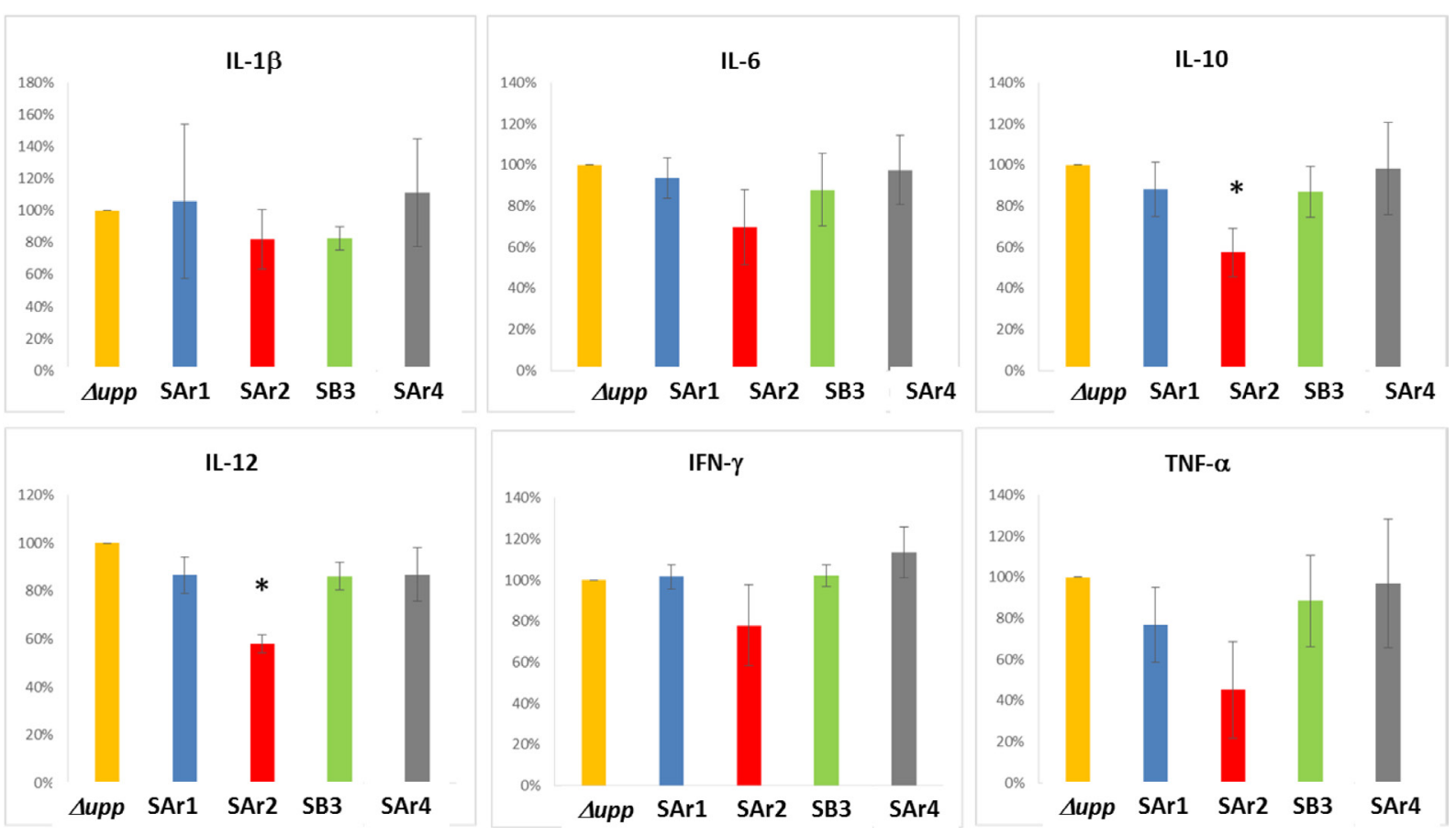

Fig. 6. In vitro cytokine production from dendritic cells (DCs) incubated with L. acidophilus cells. Immature DCs were incubated with heat-killed $L$. acidophilus cells expressing (recombinant) SlpA. Concentrations of the indicated cytokines in the supernatants of the DCs were determined using Bio-Plex system. Data are the means of values relative to the cytokine levels in DC culture with the Aupp strain from two independent experiments performed in duplicate. Error bars indicate the standard deviation. Statistical significance between the data of the parent strain and each mutant was calculated using 2-way ANOVA and the post hoc paired t-test with Bonferroni correction. ${ }^{*} \mathrm{p}<0.05$.

by changing SLAP patterns on the surface of the cells. SLPs and SLAPs might function as barriers against detergents, alcohols or salts, all of which are known to have an influence on cell envelope integrity [35]. Interestingly, stress sensitivity depends on the specific SLP expressed, namely SlpA or SlpB/X. Stress tolerance is also dependent on the position of the c-myc epitope in SlpA. Expression of SlpB/X or recombinant SlpA instead of wild-type SlpA could change the lattice monolayer structure of self-assembled SLPs, which could affect the robustness of SLP assembly, the strength of its binding to the cells, the barrier function, and/or the pattern and amount of SLAPs. One or a combination of these presumed changes might affect the bacterial stress tolerance comprehensively. The tertiary and quaternary structures of SLPs are not known. A better understanding of these structures and the molecular mechanisms of stress tolerance functions of SLPs and SLAPs should allow for the development of highly stress-tolerant strains.

Binding of the host proteins uromodulin and DC-SIGN by $L$. acidophilus has been postulated to take place via SlpA [19, 24]. Therefore, it was anticipated that the ability to bind these proteins of the strains expressing SlpA versions with a surface-exposed c-myc epitope, SAr1 and SAr2, might have changed. Here we showed that the binding of uromodulin and DC-SIGN is not only altered in strains SAr1 and SAr2 but is also altered in strain SAr4, producing an SlpA variant with a non-surface-exposed c-myc epitope. This suggests that this recombinant version of SlpA might still affect the structure of the SLP monolayer and/ or the pattern of SLAPs in such a way as to result in an altered binding of these host proteins. The binding of DC-SIGN and uromodulin to strain SAr1 was decreased to $79 \%$ and $50 \%$ of that of the parent strain, respectively, and this was quite similar to the decrease in binding to strain SAr4 (70\% in each case). Whether these alterations in binding are a direct effect on possible contact sites between the human proteins and SlpA or due to changes in the general structure of the surface layer will need further clarification. The in vitro binding of DC-SIGN to the SlpB- and SlpX-expressing strain SB3 did not change, but binding was significantly increased for uromodulin, suggesting that the binding of the 2 host proteins to surface layer proteins is not specific to SlpA. The 2 host proteins likely bind to SlpB and/ or SlpX, the SLPs expressed instead of SlpA in strain SB3. Our data also suggest that more uromodulin binds to SlpB or SlpX than to SlpA and that the parts of surface layer proteins that make contact with the 2 host proteins are different. Strain SB3 might be efficiently incorporated into $\mathrm{M}$ cells via its binding ability to uromodulin, which could lead to effective modification of the host immune system. If proteins such as SlpB or SlpX allow for the producer cells to be efficiently incorporated into $\mathrm{M}$ cells, they might be applied for the future development of oral immunemodulating vehicles, such as recombinant bacteria, microcapsules or liposomes carrying these proteins on the surface. Whether SlpB and/or SlpX can bind to uromodulin strongly enough for the proteins to be efficiently delivered through $\mathrm{M}$ cells, which could lead to further efficient immune-modulation strategies, still needs to be elucidated.

Various reports exist on factors in L. acidophilus modifying the host immune system. A lipoteichoic acid (LTA)-deficient $L$. acidophilus mutant, obtained by deleting the phosphoglycerol transferase gene LBA0447, reduced IL-12 and enhanced IL-10 production in DCs, resulting in anti-inflammatory signaling 
[36]. L. acidophilus LTA is thought to be one of the main factors triggering pro-inflammatory cytokines such as IL-12. SlpA is another important factor in host immune modification; oral administration of purified SlpA from L. acidophilus protected against colitis in a mouse model by inducing regulatory signaling [23]. SlpA binds to uromodulin on M cells, leading to effective delivery of bacteria to DCs [24]. SlpA also binds to one of the C-type lectins on DCs (murine SIGNR3 and human DC-SIGN) to exert regulatory signals $[19,23]$. In addition, studies using gene knockout have implicated the surface proteins PrtX, SlpX and Lba-1029 of L. acidophilus in immune modification [16, 25, 37]. Co-culture of DCs and L. acidophilus $\triangle p r t X$, the double mutant $\triangle s \operatorname{lp} X \triangle s \operatorname{lpB}$ or strain $\triangle L b a-1029$ led to an increase in IL-10 and IL-10/IL-12 ratio, an increase in TNF- $\alpha$ or a decrease in TNF- $\alpha$ production relative to the parent strain, respectively. Our data showed that L. acidophilus strain SAr2 led to a decrease in IL-10 and IL-12 production when it was co-cultured with DCs. These results indicate that a recombinant form of SlpA, SlpAr2, might change DC signaling. In a previous report, an SlpB expressing an $L$. acidophilus mutant derived from strain NCFM showed decreased binding to DC-SIGN and led to increased production of pro-inflammatory cytokines such as IL-12p70, TNF $\alpha$ and IL-1 $\beta$ from co-cultured DCs [19]. We did not detect decreased binding of DC-SIGN to strain SB3 expressing SlpB, nor did we detect increased pro-inflammatory cytokine production from DCs cocultured with the mutant. The amounts and patterns of SlpX and SLAPs might be different between the SlpB-expressing NCFM strain and our L-92-derived strain SB3, and this could have led to the differences in binding and DC signaling. The changes in cytokine production in DCs co-cultured with the recombinant L. acidophilus strains presented here might have been caused directly by the SLP changes or indirectly via SLAPs, which might have been affected by changes in SLPs, as described above.

\section{ACKNOWLEDGEMENT}

We thank Dr. Takashi Kanaya and Dr. Hiroshi Ohno (RIKEN Center for Integrative Medical Sciences) for kindly providing an expression vector for mUmod-Fc.

\section{REFERENCES}

1. Altermann E, Russell WM, Azcarate-Peril MA, Barrangou R, Buck BL, McAuliffe O, Souther N, Dobson A, Duong T, Callanan M, Lick S, Hamrick A, Cano R, Klaenhammer TR. 2005. Complete genome sequence of the probiotic lactic acid bacterium Lactobacillus acidophilus NCFM. Proc Natl Acad Sci USA 102: 3906-3912. [Medline] [CrossRef]

2. Sanders ME, Klaenhammer TR. 2001. Invited review: the scientific basis of Lactobacillus acidophilus NCFM functionality as a probiotic. J Dairy Sci 84: 319-331. [Medline] [CrossRef]

3. Ishida Y, Bandou I, Kanzato H, Yamamoto N. 2003. Decrease in ovalbumin specific $\mathrm{IgE}$ of mice serum after oral uptake of lactic acid bacteria. Biosci Biotechnol Biochem 67: 951-957. [Medline] [CrossRef]

4. Torii S, Torii A, Itoh K, Urisu A, Terada A, Fujisawa T, Yamada K, Suzuki H, Ishida Y, Nakamura F, Kanzato H, Sawada D, Nonaka A, Hatanaka M, Fujiwara S. 2011. Effects of oral administration of Lactobacillus acidophilus L-92 on the symptoms and serum markers of atopic dermatitis in children. Int Arch Allergy Immunol 154: 236-245. [Medline] [CrossRef]

5. Inoue Y, Kambara T, Murata N, Komori-Yamaguchi J, Matsukura S, Takahashi Y, Ikezawa Z, Aihara M. 2014. Effects of oral administration of Lactobacillus acidophilus L-92 on the symptoms and serum cytokines of atopic dermatitis in Japanese adults: a double-blind, randomized, clinical trial. Int Arch Allergy Immunol 165: 247-254. [Medline] [CrossRef]

6. Shah MM, Miyamoto Y, Yamada Y, Yamashita H, Tanaka H, Ezaki T, Nagai H, Inagaki N. 2010. Orally supplemented Lactobacillus acidophilus strain L-92 inhibits passive and active cutaneous anaphylaxis as well as 2,4-dinitroflurobenzene and mite fecal antigen induced atopic dermatitis-like skin lesions in mice. Microbiol Immunol 54: 523-533. [Medline] [CrossRef]

7. Ishida Y, Nakamura F, Kanzato H, Sawada D, Yamamoto N, Kagata H, Oh-Ida M, Takeuchi H, Fujiwara S. 2005. Effect of milk fermented with Lactobacillus acidophilus strain L-92 on symptoms of Japanese cedar pollen allergy: a randomized placebocontrolled trial. Biosci Biotechnol Biochem 69: 1652-1660. [Medline] [CrossRef]

8. Ishida Y, Nakamura F, Kanzato H, Sawada D, Hirata H, Nishimura A, Kajimoto O, Fujiwara S. 2005. Clinical effects of Lactobacillus acidophilus strain L-92 on perennial allergic rhinitis: a double-blind, placebo-controlled study. J Dairy Sci 88: 527-533. [Medline] [CrossRef]

9. Goto H, Sagitani A, Ashida N, Kato S, Hirota T, Shinoda T, Yamamoto N. 2013. Anti-influenza virus effects of both live and non-live Lactobacillus acidophilus L-92 accompanied by the activation of innate immunity. Br J Nutr 110: 1810-1818. [Medline] [CrossRef]

10. Suda Y, Nagatomo M, Yokoyama R, Ohzono M, Aoyama K, Zhang X, Nakajima K, Murakami N, Shinoda T, Hirota T, Yanagihara S, Nishi JI. 2015. Highly sensitive detection of influenza virus in saliva by real-time PCR method using sugar chainimmobilized gold nanoparticles; application to clinical studies. Biotechnol Rep (Amst) 7: 64-71. [Medline] [CrossRef]

11. Kanzato H, Fujiwara S, Ise W, Kaminogawa S, Sato R, Hachimura S. 2008 Lactobacillus acidophilus strain L-92 induces apoptosis of antigen-stimulated T cells by modulating dendritic cell function. Immunobiology 213: 399-408. [Medline] [CrossRef]

12. Shah MM, Saio M, Yamashita H, Tanaka H, Takami T, Ezaki T, Inagaki N. 2012. Lactobacillus acidophilus strain L-92 induces CD4(+)CD25(+)Foxp3(+) regulatory $\mathrm{T}$ cells and suppresses allergic contact dermatitis. Biol Pharm Bull 35: 612-616. [Medline] [CrossRef]

13. Torii A, Torii S, Fujiwara S, Tanaka H, Inagaki N, Nagai H. 2007. Lactobacillus acidophilus strain L-92 regulates the production of Th1 cytokine as well as Th2 cytokines. Allergol Int 56: 293-301. [Medline] [CrossRef]

14. Hynönen U, Palva A. 2013. Lactobacillus surface layer proteins: structure, function and applications. Appl Microbiol Biotechnol 97: 5225-5243. [Medline] [CrossRef]

15. Sleytr UB, Schuster B, Egelseer EM, Pum D. 2014. S-layers: principles and applications. FEMS Microbiol Rev 38: 823-864. [Medline] [CrossRef]

16. Johnson B, Selle K, O'Flaherty S, Goh YJ, Klaenhammer T. 2013. Identification of extracellular surface-layer associated proteins in Lactobacillus acidophilus NCFM. Microbiology (Reading) 159: 2269-2282. [Medline] [CrossRef]

17. Boot HJ, Kolen CP, Pouwels PH. 1995. Identification, cloning, and nucleotide sequence of a silent S-layer protein gene of Lactobacillus acidophilus ATCC 4356 which has extensive similarity with the S-layer protein gene of this species. J Bacteriol 177 7222-7230. [Medline] [CrossRef]

18. Goh YJ, Azcárate-Peril MA, O’Flaherty S, Durmaz E, Valence F, Jardin J, Lortal S, Klaenhammer TR. 2009. Development and application of a upp-based counterselective gene replacement system for the study of the S-layer protein SlpX of Lactobacillus acidophilus NCFM. Appl Environ Microbiol 75: 3093-3105. [Medline] [CrossRef]

19. Konstantinov SR, Smidt H, de Vos WM, Bruijns SC, Singh SK, Valence F, Molle D, Lortal S, Altermann E, Klaenhammer TR, van Kooyk Y. 2008. S layer protein A of Lactobacillus acidophilus NCFM regulates immature dendritic cell and T cell functions. Proc Natl Acad Sci USA 105: 19474-19479. [Medline] [CrossRef]

20. Boot HJ, Kolen CP, Andreadaki FJ, Leer RJ, Pouwels PH. 1996. The Lactobacillus acidophilus $\mathrm{S}$-layer protein gene expression site comprises two consensus promoter sequences, one of which directs transcription of stable mRNA. J Bacteriol 178 : 5388-5394. [Medline] [CrossRef]

21. Ashida N, Yanagihara S, Shinoda T, Yamamoto N. 2011. Characterization of adhesive molecule with affinity to Caco-2 cells in Lactobacillus acidophilus by proteome analysis. J Biosci Bioeng 112: 333-337. [Medline] [CrossRef]

22. Yanagihara S, Kato S, Ashida N, Yamamoto N. 2015. Lactobacillus acidophilus CP23 with weak immunomodulatory activity lacks anchoring structure for surface layer protein. J Biosci Bioeng 119: 521-525. [Medline] [CrossRef]

23. Lightfoot YL, Selle K, Yang T, Goh YJ, Sahay B, Zadeh M, Owen JL, Colliou N, Li E, Johannssen T, Lepenies B, Klaenhammer TR, Mohamadzadeh M. 2015. SIGNR3dependent immune regulation by Lactobacillus acidophilus surface layer protein $\mathrm{A}$ in colitis. EMBO J 34: 881-895. [Medline] [CrossRef]

24. Yanagihara S, Kanaya T, Fukuda S, Nakato G, Hanazato M, Wu XR, Yamamoto N Ohno H. 2017. Uromodulin-SlpA binding dictates Lactobacillus acidophilus uptake by intestinal epithelial M cells. Int Immunol 29: 357-363. [Medline] [CrossRef]

25. Johnson BR, O'Flaherty S, Goh YJ, Carroll I, Barrangou R, Klaenhammer TR. 2017. The S-layer associated serine protease homolog PrtX impacts cell surface-mediated microbe-host interactions of Lactobacillus acidophilus NCFM. Front Microbiol 8: 1185. [Medline] [CrossRef]

26. Hymes JP, Johnson BR, Barrangou R, Klaenhammer TR. 2016. Functional analysis of an S-layer-associated fibronectin-binding protein in Lactobacillus acidophilus NCFM. Appl Environ Microbiol 82: 2676-2685. [Medline] [CrossRef]

27. Schägger H. 2006. Tricine-SDS-PAGE. Nat Protoc 1: 16-22. [Medline] [CrossRef]

28. Matsuzawa A, Kanno S, Nakayama M, Mochiduki H, Wei L, Shimaoka T, Furukawa Y, Kato K, Shibata S, Yasui A, Ishioka C, Chiba N. 2014. The BRCA1/BARD1- 
interacting protein OLA1 functions in centrosome regulation. Mol Cell 53: 101-114. [Medline] [CrossRef]

29. Smit E, Jager D, Martinez B, Tielen FJ, Pouwels PH. 2002. Structural and functional analysis of the S-layer protein crystallisation domain of Lactobacillus acidophilus ATCC 4356: evidence for protein-protein interaction of two subdomains. J Mol Biol 324: 953-964. [Medline] [CrossRef]

30. Kajikawa A, Nordone SK, Zhang L, Stoeker LL, LaVoy AS, Klaenhammer TR, Dean GA. 2011. Dissimilar properties of two recombinant Lactobacillus acidophilus strains displaying Salmonella FliC with different anchoring motifs. Appl Environ Microbiol 77: 6587-6596. [Medline] [CrossRef]

31. Pulendran B, Dillon S, Joseph C, Curiel T, Banchereau J, Mohamadzadeh M. 2004. Dendritic cells generated in the presence of GM-CSF plus IL-15 prime potent CD8Tc1 responses in vivo. Eur J Immunol 34: 66-73. [Medline] [CrossRef]

32. Smit E, Oling F, Demel R, Martinez B, Pouwels PH. 2001. The S-layer protein of Lactobacillus acidophilus ATCC 4356: identification and characterisation of domains responsible for S-protein assembly and cell wall binding. J Mol Biol 305: 245-257. [Medline] [CrossRef]
33. Boot HJ, Kolen CP, Pouwels PH. 1996. Interchange of the active and silent S-layer protein genes of Lactobacillus acidophilus by inversion of the chromosomal slp segment. Mol Microbiol 21: 799-809. [Medline] [CrossRef]

34. Goh YJ, Klaenhammer TR. 2010. Functional roles of aggregation-promoting-like factor in stress tolerance and adherence of Lactobacillus acidophilus NCFM. Appl Environ Microbiol 76: 5005-5012. [Medline] [CrossRef]

35. Jordan S, Hutchings MI, Mascher T. 2008. Cell envelope stress response in Grampositive bacteria. FEMS Microbiol Rev 32: 107-146. [Medline] [CrossRef]

36. Mohamadzadeh M, Pfeiler EA, Brown JB, Zadeh M, Gramarossa M, Managlia E, Bere P, Sarraj B, Khan MW, Pakanati KC, Ansari MJ, O'Flaherty S, Barrett T, Klaenhammer TR. 2011. Regulation of induced colonic inflammation by Lactobacillus acidophilus deficient in lipoteichoic acid. Proc Natl Acad Sci USA 108 Suppl 1: 4623-4630. [Medline] [CrossRef]

37. Zadeh M, Khan MW, Goh YJ, Selle K, Owen JL, Klaenhammer T, Mohamadzadeh M. 2012. Induction of intestinal pro-inflammatory immune responses by lipoteichoic acid. J Inflamm (Lond) 9: 7. [Medline] [CrossRef] 